Y. Z. Flicker

Nagoya Math. J.

Vol. 106 (1987), 121-142

\title{
STABLE BASE CHANGE FOR SPHERICAL FUNCTIONS
}

\author{
YUVAL Z. FLICKER
}

\section{To the memory of Takuro Shintani}

\section{§. Introduction}

Let $E / F$ be an unramified cyclic extension of local non-archimedean fields, $G$ a connected reductive group over $F, K(F)$ (resp. $K(E)$ ) a hyperspecial maximal compact subgroup of $G(F)$ (resp. $G(E)$ ), and $H(F)$ (resp. $H(E)$ ) the Hecke convolution algebra of compactly-supported complexvalued $K(F)$ (resp. $K(E)$ )-biinvariant functions on $G(F)$ (resp. $G(E)$ ). Then the theory of the Satake transform defines (see $\S 2$ ) a natural homomorphism $H(E) \rightarrow H(F), \phi \rightarrow f$. There is a norm map $N$ from the set of stable twisted conjugacy classes in $G(E)$ to the set of stable conjugacy classes in $G(F)$; it is an injection (see [Ko]). Let $\Phi^{\prime}(x, f)$ denote the stable orbital integral of $f$ in $H(F)$ at the class $x$, and $\Phi^{\prime}(y, \phi)$ the stable twisted orbital integral of $\phi$ in $H(E)$ at the class $y$. Note that the set of regular elements in $G(F)$ is dense, and the orbital integral $\Phi(x, f)$ is uniquely determined by its restriction to the regular set. We prove

Theorem. If $\phi$ maps to $f$, then $\Phi^{\prime}(x, \phi)=\Phi^{\prime}(N x, f)$ for any twisted stable conjugacy class $x$ in $G(E)$ with a norm $N x$ regular in $G(F)$, and $\Phi^{\prime}(y, f)=0$ for any regular class $y$ in $G(F)$ which is not of the form $N x$.

This Theorem, which is sometimes called the Fundamental Lemma, is important for the study of the Saito-Shintani base-change lifting of automorphic forms using the trace formula. In the special case where $G$ is the unitary group $U(3)$ in three variables the Theorem becomes Lemma 3.3 of [UP], which we used in [UA]. The Theorem has obvious applications also to the study of both base-change and endoscopic liftings in the context of general unitary groups, as suggested by the work of [UP], [UA] in the case of $U(3)$, as well as by [U(2)]. In particular, it is used in

Received February 24, 1986.

Revised September 18, 1986.

Partially supported by an NSF grant. 
[Rig; IV] to study the quadratic base-change lifting form $U(n, E / F)$ to $G L(n, E)$ for automorphic representation with a square-integrable component at a finite place of $F$ which splits in $E$.

The proof of this local Theorem is based on an application of the global trace formula; it is inspired by Kazhdan's proof ([K], Appendix) of his density theorem. We reduce the Theorem to the case (due to Kottwitz) where $\phi$ and $f$ are the unit elements $\phi^{0}$ and $f^{0}$ of the Hecke algebras $H(E)$ and $H(F)$, on using a new type of functions, which we call "regular" functions. These are essentially elements in the Hecke algebra with respect to an Iwahori subgroup; they are not spherical functions. They have the properties that (i) they are supported on the split regular set, (ii) they annihilate the $G$-modules without Iwahori fixed vectors. For example, when $G=S L(2)$, for each $n \geq 1$ there is a regular function $f=f_{n}$; it has the property that its normalized orbital integral $F(g, f)$ is zero unless $g$ is conjugate to $\left(\begin{array}{cc}a & 0 \\ 0 & a^{-1}\end{array}\right)$ with $|a|=q^{n}$, where $F\left(\left(\begin{array}{cc}a & 0 \\ 0 & a^{-1}\end{array}\right), f_{n}\right)=1$.

Our approach is new even in the case of base-change for $G L(2)$, and is considerably simpler than all previous approaches. As is well known, the Theorem was first proven by Saito for $G L(2)$, and recently by ArthurClozel (manuscript in preparation) for $G L(n)$. This last technique is also suggested by the proof of $[\mathrm{K}]$, Appendix, but it is based on usage of spherical functions, and relies on a deep result of [BZ'] asserting that when $G=G L(n)$ the only tempered elliptic $G$-modules which are subquotients of unramified principal series representations are Steinberg. An analogous description is known for $G=U(3)$, and as noted in [UP] (e.g. p. 132, l. 17/18) and [UA], we checked that the technique of spherical functions can be used to prove the Theorem when $G=U(3)$. However we could not prove the Theorem for other unitary groups $G=U(n)(n>3)$ using this technique. This motivated us to develop our method, which does not require analogous facts concerning composition factors of unramified principal series representations, hence makes it possible to prove the Theorem for an arbitrary reductive group.

Our method applies quite generally: it is used in the case of the metaplectic correspondence [FK] (where non-algebraic groups appear), and in the case of the symmetric square [Sym; II], which is not a base-change situation. The technique is likely to apply in any lifting situation to reduce the transfer of orbital integrals of spherical functions to that of the unit elements in the Hecke algebras. To simplify the exposition we 
deal here only with the case of stable base-change. Unstable analogues of the Theorem are known in some cases of arbitrary rank; see [ $\left.\mathrm{K}^{\prime}\right]$ and [GL(n)], and [Sym; V]. Also, it will be easier for the reader to (follow the author and) read the proofs first in the context of the most important cases, namely when $G$ is split (easiest case is $G=G L(n)$ ), or when $G^{\prime}=$ $G L(n, E)$ and $G$ is the unitary group $U(n, E / F)$ defined using a quadratic extension $E / F$.

Regular functions play a crucial role not only in the study of spherical functions, but also in the study of analytical aspects of the trace formula and its applications to lifting problems. Regular functions afford establishing the metaplectic correspondence in [FK], the simple algebra correspondence in [Rig; III], and base-change lifting for unitary groups in [Rig; IV], without having to study the orbital integrals of the non ellipticregular classes with appear in the trace formula. They are used in [STF] to extend the global theorems of [FK], [Rig; III] to the context of cuspidal representations with a single supercuspidal component. In the context of groups of rank one, the usage of regular functions affords a simple proof for the comparison of trace formulae for general test functions, needed to establish lifting theorems, bypassing the analytical difficulties encountered if no care is taken to optimize the choice of a test function. In particular, regular functions make it possible to give in [RTF] an elementary completion of the Saito-Shintani proof of the cyclic base-change theory for $G L(2)$, and to complete in [Sym; VI] the study of the symmetricsquare lifting from $S L(2)$ to $P G L(3)$ for all automorphic representations.

\section{$\S 1$. Base-change}

Let $F$ be a local or global field. Let $G^{\prime \prime}$ be a reductive group defined over $F$. Denote by $G^{\prime}$ its connected component of the identity. Suppose that $\boldsymbol{G}^{\prime \prime} / \boldsymbol{G}^{\prime}$ is cyclic, generated by $\tilde{\sigma}$. Suppose that $\boldsymbol{G}^{\prime}$ is quasisplit, and contains a Borel subgroup $\boldsymbol{B}^{\prime}$ defined over $F$ which is $\tilde{\sigma}$-invariant (thus $\tilde{\sigma} \boldsymbol{B}^{\prime}=\boldsymbol{B}^{\prime} \tilde{\sigma}$ ). For any field extension $F^{\prime}$ of $F$, put $G^{\prime \prime}\left(F^{\prime}\right)$ for the group of $F^{\prime}$ points of $G^{\prime \prime}$. Put $G^{\prime \prime}=G^{\prime \prime}(F), B^{\prime}=B^{\prime}(F)$, etc. Let $A^{\prime}$ be a maximal torus defined over $F$ in a Levi subgroup of $\boldsymbol{B}^{\prime}$; clearly $\boldsymbol{A}^{\prime}$ can be assumed to be $\tilde{\sigma}$-invariant. When $F$ is local, denote by $R$ the ring of integers of $F$. Suppose that $G^{\prime}$ and $A^{\prime}$ are defined over $R$, and that $K^{\prime}=$ $G^{\prime}(R)$ is a hyperspecial (see [Ti]) maximal compact subgroup of $G^{\prime}$ with $G^{\prime}=K^{\prime} B^{\prime}=K^{\prime} A^{\prime} K^{\prime}$. Further assume that $A^{\prime} \cap K^{\prime}=A^{\prime}(R)$. It is clear 
that such a choice of $A^{\prime}, K^{\prime}$ is possible in the case which we now discuss.

Consider the case of base-change. Let $E / F$ be a cyclic field extension, $\boldsymbol{G}$ a quasi-split reductive connected $F$-group, and $\boldsymbol{G}^{\prime}=\operatorname{Res}_{E / F} \boldsymbol{G}$ the $F$-group obtained from $G$ on restricting scalars from $E$ to $F$. Let $\tilde{F}$ be a galois extension of $F$ which contains $E$ and splits $G$. Then $G^{\prime}$ is isomorphic over $\tilde{F}$ to the product $\boldsymbol{G} \times \cdots \times \boldsymbol{G}$ of $e=[E: F]$ copies of $\boldsymbol{G}$, on which $\operatorname{Gal}(\tilde{F} / F)$ acts through its quotient $\operatorname{Gal}(E / F)$, as follows. Fix $\sigma$ in $\operatorname{Gal}(\tilde{F} / F)$ whose restriction to $E$ generates $\operatorname{Gal}(E / F)$. Write $\tilde{\sigma}\left(x_{1}, \cdots, x_{e}\right)=\left(x_{2}, \cdots\right.$, $\left.x_{e}, x_{1}\right)$. Then $\operatorname{Gal}(\tilde{F} / F)$ acts on $G(\tilde{F})$ by $\tau: x \rightarrow \tau x$, and on $G^{\prime}(\tilde{F})$ by

$$
\tau\left(x_{1}, \cdots, x_{e}\right)=\tilde{\sigma}^{i}\left(\tau x_{1}, \cdots, \tau x_{e}\right)
$$

if the restriction of $\tau$ to $E$ coincides with $\sigma^{i}(0 \leq i<e)$. Hence $G^{\prime}(E)=$ $G(E) \times \cdots \times G(E)$, and

$$
G^{\prime}=G^{\prime}(F)=\left\{\left(x, \sigma x, \cdots, \sigma^{e-1} x\right) ; \quad x \text { in } G(E)\right\}
$$

is isomorphic to $G(E)$. Put $\boldsymbol{G}^{\prime \prime}=\boldsymbol{G}^{\prime} \times\langle\tilde{\sigma}\rangle$. Fix a Borel subgroup $\boldsymbol{B}$ in $\boldsymbol{G}$ and a maximally split torus $A$ in $B$, defined over $F$. Put $A=A(F)$, $A^{\prime}=\operatorname{Res}_{E / F} A$; then $A^{\prime}=A^{\prime}(F)$ is isomorpihc to $A(E)$. Similarly we have $B, B^{\prime}$. We assume that $\boldsymbol{G}, \boldsymbol{B}, \boldsymbol{A}$ are defined over $R$ when $F$ is local.

Let $\hat{G}^{0}$ be a complex reductive connected group whose root datum $\left(X, \Delta, X^{*}, \Delta^{\vee}\right)$ is dual to that of $G$ (see [B], where $\hat{G}^{0}$ is denoted by ${ }^{L} G^{0}$ ). Put $\hat{G}$ for the semi-direct product $\hat{G}^{0} \times \operatorname{Gal}(\tilde{F} / F)$ (see [B]). Write $\hat{G}^{\prime 0}$ for $\hat{G}^{0} \times \cdots \times \hat{G}^{0}$, and $\hat{G}^{\prime \prime}$ for $\hat{G}^{\prime 0} \times \operatorname{Gal}(\tilde{F} / F)$ where $\operatorname{Gal}(\tilde{F} / F)$ acts by the formula of $(*)$. In particular $\hat{G}^{0}$ is endowed with a $\operatorname{Gal}(\tilde{F} / F)$-invariant maximal torus $\hat{A}^{0}$; we put $\hat{A}$ for the subgroup $\hat{A}^{0} \times \operatorname{Gal}(\tilde{F} / F)$ of $\hat{G}$. $\hat{A}^{\prime}$ $=\hat{A}^{0} \times \cdots \times \hat{A}^{0}$ is a maximal torus in $\hat{G}^{\prime 0}$; we put $\hat{A}^{\prime \prime}$ for the subgroup $\hat{A}^{\prime} \times \operatorname{Gal}(\tilde{F} / F)$ of $\hat{G}^{\prime \prime}$.

Suppose now that $F$ is local non-archimedean, $E / F$ is unramified, and $G$ splits over a finite unramified extension $\tilde{F}$ of $F$. We assume that $\tilde{F}$ contains $E$. Fix a generator $\sigma$ of $\operatorname{Gal}(\tilde{F} / F)$. Its restriction to $E$ generates Gal $(E / F)$. Let $X^{*}(\hat{A})$ (resp. $X^{*}\left(\hat{A}^{\prime \prime}\right)$ ) be the group of rational characters $\chi$ in $X^{*}\left(\hat{A}^{0}\right)$ (resp. $X^{*}\left(\hat{A}^{\prime}\right)$ ) with $\chi=\chi \circ \tau$ for all $\tau$ in $\operatorname{Gal}(\tilde{F} / F)$. Then $X^{*}(\hat{A}) \simeq Z^{r}, X^{*}\left(\hat{A}^{\prime \prime}\right) \simeq Z^{r \prime}$, with $r \leq r^{\prime}$. We have $A^{\prime} \mid A^{\prime}(R) \simeq X^{*}\left(\hat{A}^{\prime \prime}\right)$ and $A / A(R) \simeq X^{*}(\hat{A})$. The norm map $N$ from $A^{\prime}$ to $A$ defined by $N a=a \sigma(a)$ $\cdots \sigma^{e-1}(a)$ defines a map from $X^{*}\left(\hat{A}^{\prime \prime}\right)$ to $X^{*}(\hat{A})$ which is dual to the diagonal embedding of $\hat{A}$ in $\hat{A}^{\prime \prime}$.

Let $C\left(G^{\prime}\right)$ be the space of complex-valued compactly-supported locally- 
constant functions on $G^{\prime \prime}$ which are supported on $G^{\prime} \times \tilde{\sigma}$. The "twisted" orbital integral of $\phi$ in $C\left(G^{\prime}\right)$ at $x$ in $G^{\prime \prime}$ is

$$
\Phi(x, \phi)=\int \phi\left(y x y^{-1}\right) d y .
$$

It is taken over $G^{\prime} \mid Z(x)$, where $Z(x)$ is the centralizer of $x$ in $G^{\prime}$, and $d y$ is a Haar measure (which is unique up to a scalar multiple (see, e.g., [BZ])). If $x$ is semi-simple in $G^{\prime \prime}$, then the orbit $\left\{y x y^{-1}\right\}$ is closed and the convergence is clear. This is the only case needed below. For convergence in general see $[\mathrm{R}]$. We say that $x$ (in $G^{\prime} \times \tilde{\sigma}$ ) is regular if its centralizer $Z(x)$ in $G^{\prime}$ is a torus. We shall also consider the integral ' $\Phi(x, \phi)$ on the set of regular $x$ in $G^{\prime} \times \tilde{\sigma}$; ' $\Phi(x, \phi)$ is defined on replacing $Z(x)$ in the definition of $\Phi(x, \phi)$ by the split component of $Z(x)$.

Let $x=s u=u s$ be the Jordan decomposition of $x$ in $G^{\prime \prime}$ into a semisimple and unipotent elements $s$ and $u$. For any algebraic group $X$, let $L(X)$ be the Lie algebra of (the connected component of) $X$. Put

$$
\Delta(x)=\Delta(s)=\left|\operatorname{det}\left\{(1-\operatorname{Ad}(s)) \mid L\left(G^{\prime \prime}\right) / L(Z(s))\right\}\right|^{1 / 2},
$$

and

$$
F(x, \phi)=\Delta(x) \Phi(x, \phi), \quad{ }^{\prime} F(x, \phi)=\Delta(x)^{\prime} \Phi(x, \phi) .
$$

A subgroup $P^{\prime \prime}$ of $G^{\prime \prime}$ is called here parabolic if $P^{\prime}=G^{\prime} \cap P^{\prime \prime}$ is a parabolic subgroup of $G^{\prime}$, and $G^{\prime \prime} / G^{\prime} \simeq P^{\prime \prime} \mid P^{\prime}$. Let $N$ be the unipotent radical of $P^{\prime \prime}$; it is equal to the unipotent radical of $P^{\prime}$. By a Levi subgroup $M^{\prime}$ of $P^{\prime}$ we mean a standard Levi subgroup, namely one which contains $A^{\prime}$. Then $P^{\prime}=M^{\prime} N$ is a Levi decomposition of $P^{\prime}$, and $P^{\prime \prime}=$ $M^{\prime \prime} N$ of $P^{\prime \prime}$, where $M^{\prime \prime}$ is a Levi subgroup of $P^{\prime \prime}$ satisfying $M^{\prime \prime} / M^{\prime} \simeq$ $P^{\prime \prime} \mid P^{\prime}$. For $m$ in $M^{\prime \prime}$, put $\delta_{P^{\prime}}(m)=|\operatorname{det} \operatorname{Ad}(m)| L(N) \mid$. Put $\delta$ for $\delta_{B^{\prime}}$.

For $\phi$ in $C\left(G^{\prime}\right)$ and a parabolic $P^{\prime}=M^{\prime} N$, define $\phi_{N}$ in $C\left(M^{\prime}\right)$ by

$$
\phi_{N}(m)=\delta_{P^{\prime}}(m)^{1 / 2} \int_{N} d n \int_{K^{\prime}} \phi\left(k^{-1} m n k\right) d k .
$$

$\phi_{N}$ depends on $N$. But its orbital integral at an element $m$ of $M^{\prime \prime}$ regular (see $\S 2$ ) in $G^{\prime \prime}$ depends only on $M^{\prime \prime}$. Indeed, a standard computation (see, e.g., [FK], § 7) shows that $F(m, \phi)=F^{M^{\prime \prime}}\left(m, \phi_{N}\right)$ for such $m$. $F^{M^{\prime \prime}}$ is the orbital integral, multiplied by the $\Delta_{M^{\prime \prime}}$-factor, with respect to $M^{\prime \prime}$.

Let $\mu$ be an unramified character of $A^{\prime}$. Namely it is trivial on $A^{\prime}(R)$. Extend $\mu$ to a character on $B^{\prime}=A^{\prime} U^{\prime}$ by setting $\mu=1$ on the unipotent radical $U^{\prime}$. Let $I^{\prime}(\mu)$ be the $G^{\prime}$-module unitarily induced from 
$\mu$. Thus $I^{\prime}(\mu)=$ Ind $\left(\delta^{1 / 2} \mu ; G^{\prime}, B^{\prime}\right)$. It is unramified. Namely it has a nonzero $K^{\prime}$-fixed vector. The dimension of the space of $K^{\prime}$-fixed vectors is one. Hence $I^{\prime}(\mu)$ has a unique irreducible unramified subquotient $\pi_{\mu}^{\prime}$. Any irreducible unramified $G^{\prime}$-module can be realized in this way (see [Ca]). The map $\mu \times \sigma \rightarrow \pi_{\mu}^{\prime}$ yields an isomorphism of sets from the variety of conjugacy classes in $\hat{G}^{\prime \prime}$ which intersect $\hat{G}^{\prime} \times \sigma$, to the set of irreducible unramified $G^{\prime}$-modules.

Given a $G^{\prime}$-module $\left(\pi^{\prime}, V\right)$, put ${ }^{\tilde{}} \pi^{\prime}(x)=\pi^{\prime}(\tilde{\sigma} x)$. Such $\pi^{\prime}$ is called $\tilde{\sigma}$ invariant if it is equivalent to ${ }^{\sigma} \pi^{\prime}$. Namely, there exists an intertwining operator $A$ with $\pi^{\prime}(\tilde{\sigma} x) A=A \pi^{\prime}(x)$ for all $x$ in $G^{\prime}$. If $\pi^{\prime}$ is irreducible, then by Schur's lemma $A^{e}$ is a scalar which we normalize to have $A^{e}=1$, and put $\pi^{\prime}(\tilde{\sigma})=A$. Hence a $\tilde{\sigma}$-invariant $G^{\prime}$-module extends to a $G^{\prime \prime}$-module. Note that $\pi_{\mu}^{\prime}$ is $\tilde{\sigma}$-invariant if and only if $I^{\prime}(\mu)$ and $\tilde{\sigma} I^{\prime}(\mu)$ have equal characters (namely equal composition series up to order).

Definition. By a strongly irreducible $G^{\prime \prime}$-module we mean here a $G^{\prime \prime}$-module whose restriction to $G^{\prime}$ is irreducible; it is an extension to $G^{\prime \prime}$ of a $\tilde{\sigma}$-invariant $G^{\prime}$-module.

Given a $G^{\prime \prime}$-module $\pi^{\prime}, \phi$ in $C\left(G^{\prime}\right)$ and a Haar measure $d^{\prime} x$ on $G^{\prime}$, we define the convolution operator $\pi^{\prime}(\phi)=\int \phi(x) \pi^{\prime}(x) d^{\prime} x$. It has finite rank, and its trace is denoted by $\operatorname{tr} \pi^{\prime}(\phi)$. The trace is equal to zero unless $\pi^{\prime}$ is strongly irreducible. By the character $\chi=\chi\left(\pi^{\prime}\right)$ we mean a complex-valued function on $G^{\prime} \times \tilde{\sigma}$ which is locally-constant on the regular set with

$$
\operatorname{tr} \pi^{\prime}(\phi)=\int \chi(x) \phi(x) d x
$$

for every $\phi$ which is supported on the regular set. It is easy to show that the character $\chi$ exists in this sense. It is also known that when char $F=0, \chi$ extends as a locally-integrable function to $G^{\prime} \times \tilde{\sigma}$, with

$$
\operatorname{tr} \pi^{\prime}(\phi)=\int \chi(x) \phi(x) d x
$$

for all $\phi$ in $C\left(G^{\prime}\right)$; but we do not need this deeper result.

\section{§. Satake transform}

Consider the situation of local base change in the notations of Section 1. Thus $R$ is the ring of integers in $F, R_{E}$ in $E ; K^{\prime}=G^{\prime}(R)=G\left(R_{E}\right)$ is a maximal compact subgroup of the group $G^{\prime} \simeq G(E)$, and $K=G(R)$ 
of $G$. Let $H^{\prime}$ be the convolution algebra of $K^{\prime}$-biinvariant compactlysupported complex-valued functions $\phi$ on $G^{\prime}$. Recall $(\S 1)$ that $A^{\prime} / A^{\prime}(R) \simeq$ $X^{*}\left(\hat{A}^{\prime \prime}\right)$. Since $\phi$ in $H^{\prime}$ is $K^{\prime}$-biinvariant, $F(a, \phi)=\phi_{U}(a)$ depends only on the projection $\lambda$ of $a$ in $X^{*}\left(\hat{A}^{\prime \prime}\right)$, and we denote it by $F(\lambda, \phi)$. Given an admissible irreducible $G^{\prime}$-module $\pi^{\prime}, \phi$ in $H^{\prime}$ and a Haar measure $d^{\prime} x$ on $G^{\prime}$, the convolution operator $\pi^{\prime}(\phi)=\int \phi(x) \pi^{\prime}(x) d^{\prime} x$ has finite rank, and its trace is denoted by $\operatorname{tr} \pi^{\prime}(\phi)$. Since $\phi$ is spherical, we have $\pi^{\prime}(\phi)=0$, unless $\pi^{\prime}$ is unramified. In the latter case $\pi^{\prime}(\phi)$ acts as a scalar multiple of the projection on the space of $K^{\prime}$-fixed vectors in the space of $\pi^{\prime}$. We have $\operatorname{tr} \pi_{\mu}^{\prime}(\phi)=\operatorname{tr} I^{\prime}(\mu, \phi)$ for $\phi$ in $H^{\prime}$. As noted in Section 1 there is a bijection from the set of conjugacy classes in $\hat{G}^{\prime \prime}$ which intersect $\hat{G}^{\prime} \times \sigma$, to the set of irreducible unramified $G^{\prime}$-modules $\pi_{\mu}^{\prime}$. Let $W=N / Z$ be the quotient of the normalizer $N=N\left(\hat{A}^{\prime} \times \sigma\right)$ of $\hat{A}^{\prime} \times \sigma$ in $\hat{G}^{\prime}$, by the centralizer $Z=$ $Z\left(\hat{A}^{\prime} \times \sigma\right)$. The Satake isomorphism $\phi \rightarrow \phi^{\vee}$ identifies $H^{\prime}$ with the algebra $C\left[\hat{A}^{\prime} \times \sigma\right]^{W}$ of $W$-invariant finite Laurent series on $\hat{A}^{\prime} \times \sigma$. It is defined by

$$
\phi^{\vee}(\mu \times \sigma)=\operatorname{tr} \pi_{\mu}^{\prime}(\phi)=\int_{A^{\prime}} \mu(a) F(a, \phi) d a=\sum_{\lambda} F(\lambda, \phi) \lambda(\mu)
$$

( $\lambda$ in $\left.X^{*}\left(\hat{A}^{\prime \prime}\right)\right)$. The first equality is our definition, the second follows from a standard computation [D] of the character of an induced representation. We put $\lambda(\mu)$ for $\mu(a)$ if a maps to $\lambda$ via $A^{\prime} / A^{\prime}(R) \simeq X\left(\hat{A}^{\prime \prime}\right)$.

Similarly, let $H$ be the Hecke algebra of $G$ with respect to $K$. The members of $H$ are denoted by $f$, and the Satake transform by $f^{\vee}$. Let $H^{\prime} \rightarrow H, \phi \rightarrow f$, be the map dual to the diagonal embedding $\hat{G} \rightarrow \hat{G}^{\prime \prime}$, by $x \times \sigma \rightarrow(x, \cdots, x) \times \sigma$. Thus $\phi$ defines $f$ by

$$
f^{\vee}(x \times \sigma)=\phi^{\vee}((x, \cdots, x) \times \sigma) \quad\left(=\phi^{\vee}((x \sigma(x) \cdots, 1, \cdots, 1) \times \sigma)\right) .
$$

Definition. $\phi$ in $H^{\prime}$ corresponds to $f$ in $H$ if $f$ is the image of $\phi$ under the above map $H^{\prime} \rightarrow H$.

If $\phi$ corresponds to $f$ then

$$
\sum_{\lambda} F(\lambda, f) \lambda(\mu)=\sum_{\lambda} F(\lambda, \phi) \lambda(\mu \sigma(\mu) \cdots),
$$

and

$$
F(a, f)=F(\alpha, f)=\sum F(\lambda, \phi) .
$$

Here $a$ in $A$ corresponds to $\alpha$ in $X^{*}(\hat{A})$, and the last sum ranges over all $\lambda$ in $X^{*}\left(\hat{A}^{\prime \prime}\right)$ with $N \lambda=\lambda+\sigma \lambda+\cdots+\sigma^{e-1} \lambda$ equals $\alpha$. If there is no $\lambda$ with $N \lambda=\alpha$ then $F(a, f)$ is zero. 
The element $\phi$ of $H^{\prime}$ defines a function, denoted again by $\phi$, in $C\left(G^{\prime}\right)$, by $\phi(x \times \tilde{\sigma})=\phi(x)$. A standard change of variable shows that the sum on the right of $\left(^{*}\right)$ is the "twisted" orbital integral $F(b \times \tilde{\sigma}, \phi)$, which is defined in Section 1 , where $b$ in $A^{\prime}$ maps to $\lambda$ in $X^{*}\left(\hat{A}^{\prime \prime}\right)$ with $N \lambda=\alpha$. We denote any $a$ in $A$ which maps to $\alpha$ by $N b$, and conclude

Proposition. If $\phi$ corresponds to $f$ under the map $H^{\prime} \rightarrow H$, then $F(x \times$ $\tilde{\sigma}, \phi)=F(N x, f)$ for all regular $x$ in $A^{\prime}$. If a in $A$ defines $\alpha$ in $X^{*}(\hat{A})$ such that $\alpha \neq N \lambda$ for all $\lambda$ in $X^{*}\left(\hat{A}^{\prime \prime}\right)$ then $F(a, f)$ is zero. Moreover, we have that $F(x, f)=0$ for any regular $x$ in $G$ which is not a norm.

The third claim follows from the second; the proof is omitted.

The unit element $f^{0}$ of $H$ (resp. $\phi^{0}$ of $H^{\prime}$ ) is supported on $K=K^{\prime} \cap G$ (resp. $K^{\prime}$ ), and attains there the value $|K|^{-1}$ (resp. $\left|K^{\prime}\right|^{-1}$ ). $|K|$ indicates the volume of $K$ with respect to the Haar measure $d x$ on $G$ fixed in the definition of the Satake transform. By restriction of scalars the differential form $\omega$ on $\boldsymbol{G}$ which defines $d x$ yields a form $\omega^{\prime}$ on $\boldsymbol{G}^{\prime}$, and a measure $d^{\prime} x$ on $G^{\prime}$, so that the volume $\left|K^{\prime}\right|$ is defined.

Corollary. $\phi^{0}$ corresponds to $f^{0}$ (under the map $H^{\prime} \rightarrow H$ ).

We use below the following definitions. Let $\bar{F}$ be an algebraic closure of $F$. The elements $x, x^{\prime}$ in $G^{\prime}$ are called (stably) $\sigma$-conjugate if there is $y$ in $G^{\prime}$ (resp. $\left.G^{\prime}(\bar{F})\right)$ with $y(x \times \tilde{\sigma})=\left(x^{\prime} \times \tilde{\sigma}\right) y$, namely $x \times \tilde{\sigma}, x^{\prime} \times \tilde{\sigma}$ are (stably) conjugate by $G^{\prime}$. There is a norm map $N$ from the set of stable $\sigma$-conjugacy classes in $G^{\prime}$, to the set of stable conjugacy classes in $G$, obtained from the map $x \rightarrow x \sigma(x) \cdots \sigma^{e-1}(x)$ from $G(E)$ to itself (see [Ko]). We say that $x$ in $G^{\prime}$ is $\sigma$-regular if $N x$ is regular in $G$, namely the centralizer $Z(N x)$ of $N x$ in $G$ is a torus. This definition is equivalent to the one given in Section 1. Indeed, the centralizers $Z(x \times \tilde{\sigma}), Z\left(x^{\prime} \times \tilde{\sigma}\right)$ in $G^{\prime}$ of $x \times \tilde{\sigma}, x^{\prime} \times \tilde{\sigma}$, where $x, x^{\prime}$ are stably $\sigma$-conjugate, are inner forms of each other, and of $Z(N x)$. Hence these centralizers are isomorphic over $\bar{F}$. They are all isomorphic tori when $x \times \tilde{\sigma}$ is regular (i.e., $x$ is $\sigma$-regular). We always use these isomorphisms to transfer differential forms, and Haar measures.

For $\phi$ in $C\left(G^{\prime}\right)$ we write $\Phi(x, \phi)$ (and in Section 3 we write $\Phi(x \sigma, \phi)$ ) for $\Phi(x \times \tilde{\sigma}, \phi)$, where no confusion should arise. Here $x$ is in $G^{\prime}$. Put $\Phi^{\prime}(x, \phi)$ for the sum $\sum \Phi\left(x^{\prime}, \phi\right)$ over a set of representatives $x^{\prime}$ for the $\sigma$ conjugacy classes within the stable $\sigma$-conjugacy class of $x$ in $G^{\prime}$. Similarly, we put $\Phi^{\prime}(x, f)$ for the sum $\sum \Phi\left(x^{\prime}, f\right)$ over a set of representatives $x^{\prime}$ for 
the conjugacy classes within the stable conjugacy class of $x$ in $G$. Put $F^{\prime}(x, \phi)=\Delta(x) \Phi^{\prime}(x, \phi), F^{\prime}(y, f)=\Delta(y) \Phi^{\prime}(y, f)$. Note that $\Delta(x \times \tilde{\sigma})=\Delta(N x)$.

Definition. $\phi$ in $C\left(G^{\prime}\right)$ and $f$ in $C(G)$ are matching, or have matching (stable) orbital integrals, if $F^{\prime}(x, \phi)=F^{\prime}(N x, f)$ for all $\sigma$-regular $x$ in $G^{\prime}$, and $\Phi^{\prime}(y, f)=0$ for any regular $y$ in $G$ which is not of the form $N x, x$ in $G^{\prime}$.

In particular, we emphasize that below we identify $\phi$ in $H^{\prime}$ with an element in $C\left(G^{\prime}\right)$ via $\phi(x \times \tilde{\sigma})=\phi(x)$, and all orbital integrals (in $\S 5$ etc.) are "twisted": $F(\lambda, \phi)$ is $F(a \times \tilde{\sigma}, \phi)$ (but not $F(a, \phi)$ ), if $a$ in $A^{\prime}$ maps to $\lambda$ in $X^{*}\left(\hat{A}^{\prime \prime}\right)$, etc. Further, $G^{\prime}=G^{\prime}(F)$ is isomorphic to $G(E)$. We prefer to use $G^{\prime}$ and $\tilde{\sigma}$, which are defined over $F$, for the Satake transform and in the study ( $(5)$ of stable conjugacy, and $G^{\prime \prime}=G(E) \times\langle\sigma\rangle$ for representation theory.

The character $\chi$, defined in Section 1 , is a function on the space of $(\sigma-)$ conjugacy classes of $(\sigma-)$ regular elements. Such function, which attains the same value on all $(\sigma-)$ conjugacy classes within any stable $\left(\sigma^{-}\right)$conjugacy class of $\left(\sigma^{-}\right)$regular elements, is called $\left(\sigma^{-}\right)$stable.

\section{§3. Unit element}

Proposition. For any $\sigma$-regular $\delta$ in $G^{\prime}=G(E)$ we have $\Phi^{\prime}\left(\delta \sigma, \phi^{0}\right)=$ $\Phi^{\prime}\left(N \delta, f^{0}\right)$, namely $\phi^{0}$ and $f^{0}$ are matching.

Proof (Kottwitz [Ko']). We may assume that $\delta$ lies in $K^{\prime}, \Phi\left(\delta \sigma, \phi^{0}\right)$ is the sum of

$$
\left|Z(\delta \sigma) \backslash Z(\delta \sigma) g K^{\prime} g^{-1}\right| /\left|K^{\prime}\right|=\left|Z(\delta \sigma) \cap g K^{\prime} g^{-1} \backslash g K^{\prime} g^{-1}\right| /\left|K^{\prime}\right|=\left|Z(\delta \sigma) \cap g K^{\prime} g^{-1}\right|^{-1}
$$

over the $g$ in $Z(\delta \sigma) \backslash G^{\prime} \mid K^{\prime}$ with $g^{-1} \delta \sigma g$ in $K^{\prime}$. Here $Z(\delta \sigma)$ is the centralizer of $\delta \sigma$ in $G^{\prime}$. Let $x_{0}$ be a point in the building $X^{\prime}=G^{\prime} x_{0}$ of $G^{\prime}$ (see [Ti]), so that the stabilizer $\operatorname{Stab}_{G^{\prime}}\left(x_{0}\right)$ of $x_{0}$ in $G^{\prime}$ is $K^{\prime}$. Then the building $X$ of $G$ is $G x_{0}$, and $K=\operatorname{Stab}_{G}\left(x_{0}\right)$. The condition $g^{-1} \delta \sigma g$ in $K^{\prime}$ can be put in the form $g^{-1} \delta \sigma g x_{0}=x_{0}$, or $\delta \sigma x=x$, where $x=g x_{0}$ is in $X^{\prime}$. The same argument applied with $E$ replaced by $F$, and $\sigma=1$, shows that given $\delta$ in $K^{\prime}$ and $\gamma=N \delta$ in $G$, in fact in $K$, it suffices to show that: there is a bijection between (1) the set of $\sigma$-conjugacy classes in the stable $\sigma$-conjugacy class of $\delta$ which intersect $K^{\prime}$, and (2) the set of conjugacy classes in the stable conjugacy class of $\gamma$ which intersect $K$, such that for matching $\delta, \gamma$ under this bijection, we have 


$$
\sum_{x}\left|\operatorname{Stab}_{Z(\gamma)}(x)\right|^{-1}=\sum_{x^{\prime}}\left|\operatorname{Stab}_{Z(\delta \sigma)}\left(x^{\prime}\right)\right|^{-1}
$$

$x$ ranges over $Z(\gamma) \backslash X$ with $\gamma x=x ; x^{\prime}$ over $Z(\delta \sigma) \backslash X^{\prime}, \delta \sigma x^{\prime}=x^{\prime}$. Note that $G^{\prime} \mid K^{\prime} \simeq X^{\prime}=G^{\prime} x_{0}$, and that $Z(\gamma) \simeq Z(\delta \sigma)$. Write $X^{\prime \delta \sigma}$ and $X^{\gamma}$ for the set of fixed points in $X^{\prime}$ and $X$ of $\delta \sigma$ and $\gamma$, respectively.

Let $\tilde{F}^{\prime}$ denote the completion of the maximal unramified extension $F^{\text {ur }}$ of $F$. Denote by $\Phi$ a Frobenius element in Gal $\left(\tilde{F}^{\prime} / F\right)$, whose restriction to $E$ is our $\sigma$. Put $\tilde{G}=G\left(\tilde{F}^{\prime}\right)$. Then $X=\tilde{G} x_{0}, X=G x_{0}, X^{\prime}=G^{\prime} x_{0}$. The semi-direct product $\tilde{G} \times\langle\Phi\rangle$ acts on the building $X$, and

$$
\boldsymbol{X}^{\langle\gamma, \Phi\rangle}=X^{\gamma}, \boldsymbol{X}^{\langle\delta \Phi, \Phi e\rangle}=X^{\prime \delta \sigma} .
$$

Suppose $\gamma$ is given. Since $\gamma \Phi=\Phi \gamma$, we have $\langle\gamma, \Phi\rangle=\left\langle\gamma \Phi, \gamma^{e-1} \Phi^{e}\right\rangle$. Let $\tilde{R}$ be the ring of integers in $\tilde{F}^{\prime}$. As the map from $G(\tilde{R})$ to itself by $g \rightarrow$ $g^{-1} \Phi^{e}(g)$ is surjective (see $[G]$ ), there is $c$ in $G(\tilde{R})$ with $\gamma^{e-1}=c^{-1} \Phi^{e}(c)$. Put $\delta=c \gamma \Phi(c)^{-1}$ (in $G(\tilde{R})$ ) to obtain that $\left\langle\gamma^{e-1} \Phi^{e}, \gamma \Phi\right\rangle=c^{-1}\left\langle\Phi^{e}, \delta \Phi\right\rangle c$, and $X^{\gamma}=$ $c^{-1} X^{\prime \delta \sigma}$. Since $\langle\gamma, \Phi\rangle$ is commutative, $\delta=\Phi^{e}(\delta)$ lies in $G^{\prime}$, hence in $K^{\prime}$.

If the stably conjugate $\gamma^{e-1}=c^{-1} \Phi^{e}(c), \gamma^{\prime e-1}=c^{-1} \Phi^{e}\left(c^{\prime}\right)$ yield $\sigma$-conjugate $\delta=c \gamma \Phi\left(c^{-1}\right), \delta^{\prime}=c^{\prime} \gamma^{\prime} \Phi\left(c^{\prime-1}\right)$, we may assume $\delta=\delta^{\prime}$ to obtain $c^{-1} c^{\prime} \gamma^{\prime} \Phi\left(c^{-1} c^{\prime}\right)^{-1}$ $=\gamma$, and $c \gamma c^{-1}=N \delta=N \delta^{\prime}=c^{\prime} \gamma^{\prime} c^{\prime-1}$; hence $\Phi\left(c^{-1} c^{\prime}\right)=c^{-1} c^{\prime}$, and $\gamma, \gamma^{\prime}$ are conjugate. Here we put $N \delta=\delta \Phi(\delta) \cdots \Phi^{e-1}(\delta)$, and below: $N^{\prime} \delta=\delta \Phi(\delta) \cdots$ $\Phi^{e-2}(\delta)$. Given $\delta$ we find $c$ with $N^{\prime} \delta=\Phi^{e}(c) \Phi^{e-1}\left(c^{-1}\right)$, and define $\gamma=c^{-1} N \delta c$. We obtain a bijection of $(\sigma-)$ conjugacy classes $\{\delta\},\{\gamma\}$, with the required properties.

\section{§4. Regular functions}

Let $F$ be a local non-archimedean field, $E$ a cyclic extension of $F, \sigma$ a generator of $\mathrm{Gal}(E / F), G^{\prime}=G(E)$ the group of $E$-points on the group $\boldsymbol{G}$ of Section 1 , and put $G^{\prime \prime}=G^{\prime} \times\langle\sigma\rangle$ as in Section 1 . Let $\left(\pi^{\prime \prime}, V\right)$ be an admissible $G^{\prime \prime}$-module of finite length, $P^{\prime \prime}=M^{\prime \prime} N$ an $F$-parabolic subgroup of $G^{\prime \prime}$ with Levi subgroup $M^{\prime \prime}$ and unipotent radical $N$. The quotient of $V$ by $\left\{\pi^{\prime \prime}(n) v-v ; v\right.$ in $V, n$ in $\left.N\right\}$ is an $M^{\prime \prime}$-module ${ }^{\prime \prime} \pi_{N}^{\prime \prime}$, since $M^{\prime \prime}$ normalizes $N$. The Jacquet module $\pi_{N}^{\prime \prime}$ of $\pi^{\prime \prime}$ with respect to $N$ (or $P^{\prime \prime}$ ) is defined to be $\delta_{P}^{-1 / 2} \pi_{N}^{\prime \prime}$. It is admissible of finite length. The functor $\pi^{\prime \prime} \rightarrow \pi_{N}^{\prime \prime}$ is exact. Let $I_{M}^{\prime \prime}(\rho)$ be the $G^{\prime \prime}$-module $\operatorname{Ind}_{P \prime \prime}^{G^{\prime \prime}}\left(\delta_{P}^{1 / 2} \rho\right)$ induced from the $P^{\prime \prime}=M^{\prime \prime} N$-module $\delta_{P^{\prime \prime}}^{1 / 2} \rho \otimes 1$. Then $\operatorname{Hom}_{G^{\prime \prime}}\left(\pi^{\prime \prime}, I_{M^{\prime \prime}}^{\prime \prime}(\rho)\right)=\operatorname{Hom}_{M^{\prime \prime}}\left(\pi_{N^{\prime}}^{\prime \prime}, \rho\right)$ for all admissible $M^{\prime \prime}$-modules $\rho$ and $G^{\prime \prime}$-modules $\pi^{\prime \prime}$. Hence $\pi_{N}^{\prime \prime} \neq 0 \mathrm{im}$ plies that $\pi^{\prime \prime}$ is a subquotient of $I_{M}^{\prime}\left(\pi_{N}^{\prime \prime}\right)$. Note that since $\pi_{N}^{\prime \prime}$ is an $M^{\prime \prime}$ - 
module, its restriction $\pi_{N}^{\prime}$ to $M^{\prime}=M^{\prime \prime} \cap G^{\prime}$ is a $\sigma$-invariant $M^{\prime}$-module. As noted at the end of Section $1, \operatorname{tr} \pi_{N}^{\prime \prime}(\phi)$ for $\phi$ in $C\left(M^{\prime}\right)$ is the sum of $\operatorname{tr} \tau(\phi)$ over the strongly irreducible $M^{\prime \prime}$-modules $\tau$ in the composition series of $\pi_{N}^{\prime \prime}$.

As in Section 1 , let $\Sigma$ be the set of positive roots of $A^{\prime}$ in $B^{\prime}$. Fix a $\sigma$-invariant cocompact lattice $L$ in $A^{\prime}$ with the property that for any $\lambda$ in $L$ and $\alpha$ in $\Sigma$ if $|\alpha(\lambda)|=1$ then $\alpha(\lambda)=1$. Note that $L \simeq A^{\prime} \mid A^{\prime}(R) \simeq$ $X^{*}\left(\hat{A}^{\prime \prime}\right)$. Put $L^{-}$for the set of $\lambda$ in $L$ with $|\alpha(\lambda)| \leq 1$ for all $\alpha$ in $\Delta$. For any semi-simple $t=t_{0} \times \sigma$ in $G^{\prime} \times \sigma$, there exists a positive integer $\ell$, and $y$ in $G^{\prime}$, so that $y t^{e \ell} y^{-1}=\lambda s$, where $\lambda$ lies in $L^{-}$, and $s$ generates a subgroup of $G^{\prime}$ whose closure is compact. Let $P_{\lambda}^{\prime}$ be the standard (containing $B^{\prime}$ ) parabolic subgroup of $G^{\prime}$ whose Levi component $M_{\lambda}^{\prime}$ is the centralizer $Z(\lambda)$ of $\lambda$ in $G^{\prime}$, and put $P_{t}^{\prime}=M_{t}^{\prime} N_{t}^{\prime}$ for $y^{-1} P_{\lambda}^{\prime} y$. We shall be interested here in the special case where $P_{t}^{\prime}$ is $\sigma$-invariant. We then put $P_{t}^{\prime \prime}=P_{t}^{\prime} \times\langle\sigma\rangle$. It is clear that our definition of $P_{t}^{\prime}$ is equivalent to that of [C].

Choosing the sequence $\left\{K_{i}^{\prime}\right\}$ of open compact subgroups form [C], Lemma 2.1 , to be $\sigma$-invariant, the proof of $[\mathrm{C}]$, Theorem 5.2, extends to the twisted case to yield

Lemma 1. Let $t=t_{0} \times \sigma$ be a regular element of $G^{\prime \prime}$ such that $P^{\prime \prime}=$ $P_{t}^{\prime \prime}=P_{t}^{\prime} \times\langle\sigma\rangle$ is a parabolic subgroup. Then $\chi\left(\pi^{\prime \prime}\right)(t)=\chi\left({ }^{\prime} \pi_{N}^{\prime \prime}\right)(t)$. Since $\Delta(t)=\Delta_{M \prime \prime}(t) \delta_{P^{\prime \prime}}(t)^{-1 / 2}$, we have $\left(\Delta \chi\left(\pi^{\prime \prime}\right)\right)(t)=\left(\Delta_{M} \chi\left(\pi_{N}^{\prime \prime}\right)\right)(t)$ for such $t$.

Here $\chi\left(\pi^{\prime \prime}\right)$ denotes the character of $\pi^{\prime \prime}$ (see Section 1 ).

Definition. (1) For $\alpha$ in $\Sigma$ and $\lambda$ in $X^{*}\left(\hat{A}^{\prime \prime}\right)\left(\cong A^{\prime} / A^{\prime}(R)\right)$ we define $\langle\alpha, \lambda\rangle$ to be $\left|\alpha\left((b \times \sigma)^{e}\right)\right|^{1 / e}$, where $b$ corresponds to $\lambda$ in $A^{\prime}$. Then $(b \times \sigma)^{e}$ lies in $A^{\prime},\langle\alpha, \lambda\rangle$ is defined, and it depends only on $N \lambda$, but not on $\lambda$ itself.

(2) An element $\lambda$ of $X^{*}(\hat{A})$, which is equal to $N \lambda^{\prime}$ for some $\lambda^{\prime}$ in $X^{*}\left(\hat{A}^{\prime \prime}\right)$, is called regular if $\left\langle\alpha, \lambda^{\prime}\right\rangle \neq 0$ for each root $\alpha$ of $A^{\prime}$, equivalently if $\langle\alpha, \lambda\rangle \neq 0$ for each root $\alpha$ of $A$. For each $t$ in $A^{\prime}$ which corresponds to $\lambda^{\prime}$ in $X^{*}\left(\hat{A}^{\prime \prime}\right)$ with regular $N \lambda^{\prime}=\lambda$, the $M_{t}^{\prime}$ of the Lemma is equal to $A^{\prime}$.

(3) Let $\mu$ be a unitary character of $A$. Let $\lambda$ be a regular element of $X^{*}(\hat{A})$. The function $\phi$ in $C\left(G^{\prime}\right)$ is called regular (and associated with $\mu, \lambda)$ if $F\left(t^{\prime} \times \sigma, \phi\right)$ is zero for all $t^{\prime}$ in $G^{\prime}$ unless $t^{\prime}$ is $\sigma$-conjugate to an element of $A^{\prime}$ which corresponds to $\lambda^{\prime}$ in $X^{*}\left(A^{\prime \prime}\right)$ with $\lambda=N \lambda^{\prime}$ in $X^{*}(\hat{A})$, where we require that $F\left(t^{\prime} \times \sigma, \phi\right)=\mu^{-1}\left(N t^{\prime}\right)$. Since the support $S$ of the function $F\left(t^{\prime} \times \sigma, \phi\right)$ of $t^{\prime}$ in $G^{\prime}$ is open and closed in $G^{\prime}$, we assume, as we 
may, that the support of $\phi$ lies in $S$. Note that $S$ is contained in the $\sigma$ conjugacy class of $A^{\prime}$ in $G^{\prime}$. Thus from now on by a regular function we mean one which is supported on $S$.

Lemma 2. If $\phi$ is regular and $\pi^{\prime}$ is a strongly irreducible $G^{\prime \prime}$-module (see Section 1), then $\operatorname{tr} \pi^{\prime}(\phi)=\int \chi(x) \phi(x) d^{\prime} x$ is equal to

$$
w(A)^{-1} \int_{N A^{\prime}}(\Delta \chi)\left(t^{\prime} \times \sigma\right) F\left(t^{\prime} \times \sigma, \phi\right) d t .
$$

Remark. Here we integrate over $t$ in $A$ but $(\Delta \chi)\left(t^{\prime} \times \sigma\right)$ is evaluated at $t^{\prime}$ in $A^{\prime}$ with $N t^{\prime}=t$. Namely we integrate over $A^{\prime} / A^{1-\sigma} . \quad w(A)$ is the order of the Weyl group $W(A)$ of $A$ in $G$.

Proof. This follows at once from the Weyl integration formula.

Lemma 1 asserts that for $t^{\prime}$ with $\left.F(t \times \sigma, \phi)\right) \neq 0$, we have $(\Delta \chi)\left(t^{\prime} \times \sigma\right)=$ $\chi\left(\pi_{U^{\prime}}^{\prime}\right)\left(t^{\prime} \times \sigma\right)$, where $U^{\prime}$ denotes the unipotent radical of the Borel subgroup $B^{\prime}$ fixed above, and $\chi\left(\pi_{U^{\prime}}^{\prime}\right)$ indicates the character of the $A^{\prime \prime}=A^{\prime} \times\langle\sigma\rangle$ module $\pi_{U^{\prime}}^{\prime}$. As noted at the end of Section $1, \chi\left(\pi_{U^{\prime}}^{\prime}\right)$ is a sum of $\sigma$-invariant characters $\eta^{\prime}$ of $A^{\prime}$, namely strongly irreducible $A^{\prime \prime}$-modules, and we write $\eta\left(N t^{\prime}\right)$ for $\eta^{\prime}\left(t^{\prime} \times \sigma\right)$. Note that $\operatorname{tr} \pi^{\prime}(\phi)$ vanishes unless for some $\eta^{\prime}$ we have $\eta\left(N t^{\prime}\right)=\mu\left(N t^{\prime}\right)$ for $t^{\prime}$ with $N t^{\prime}$ in $A(R)$. In the latter case $\operatorname{tr} \pi^{\prime}(\phi)$ is equal to

$$
w(A)^{-1} \sum_{\eta^{\prime}} \int_{N A^{\prime}} \eta\left(N t^{\prime}\right) \mu\left(N t^{\prime}\right)\left|F\left(t^{\prime} \times \sigma, \phi\right)\right| d t=\sum_{\eta^{\prime}} \eta(\lambda) \mu(\lambda) \int_{N A^{\prime}(R)} \eta\left(N u^{\prime}\right) \bar{\mu}\left(N u^{\prime}\right) d u .
$$

Here $\eta^{\prime}$ ranges over the set of characters in $\chi\left(\pi_{U^{\prime}}^{\prime}\right)$. The sum ranges only over the $\eta^{\prime}$ for which the integral on the right is non-zero, hence equal to one (for a suitable choice of measure on $A(R)$ ). For such $\eta^{\prime}$ the expression $\eta\left(N t^{\prime}\right) \mu\left(N t^{\prime}\right)$ depends only on $\lambda=N \lambda^{\prime}$, and is denoted by $\eta(\lambda) \mu(\lambda)$. We write $\lambda(\eta)$ for this expression, where now $\eta$ indicates the element of $\hat{A}=X^{*}$ $\left(X^{*}(\hat{A})\right)$ defined by $\lambda \rightarrow \eta(\lambda) \mu(\lambda)$ for a varying $\lambda$. In these notations we conclude that we have the following

Lemma 3. Fix $\eta^{\prime}$ in $\chi\left(\pi_{U^{\prime}}^{\prime}\right)$; then there is a subset $W\left(\pi_{U^{\prime}}^{\prime}\right)$ of $W(A)$, depending on $\pi_{U^{\prime}}^{\prime}$ and $\eta^{\prime}$, such that

$$
\operatorname{tr} \pi^{\prime}(\phi)=\sum_{w} \lambda(w \eta)
$$

The sum is over $w$ in $W\left(\pi_{U^{\prime}}^{\prime}\right)$, and $w \eta$ is the element of $\hat{A}$ obtained under the action of $w$. It is clear from Frobenius reciprocity that the 
$\sigma$-invariant $G^{\prime}$-module $\pi^{\prime}$ is a subquotient of the $G^{\prime}$-module unitarily induced from the character $a \rightarrow \eta^{\prime}(a \times \sigma)=\eta(N a)$ of $A^{\prime}$, extended trivially to $B^{\prime}$. In particular, we have the following.

LEMMA 4. If $\mu$ is unramified then $\eta^{\prime}$ is unramified, and $\pi^{\prime}$ is a subquotient of the $G^{\prime}$-module $I\left(\eta^{\prime}\right)$ induced from an unramified character of the Borel subgroup. Hence $\pi^{\prime}$ has a vector fixed by the action of an Iwahori subgroup.

Proof. The last assertion follows from [B'], Lemma 4.7.

In the special case where $E=F, \sigma=1, G^{\prime \prime}=G^{\prime}=G$, our definitions become the following

Definition. The function $f$ in $C(G)$ is called regular (and associated with $\mu, \lambda)$ if $F(t, f)$ is zero for all $t$ in $G$ unless $t$ is conjugate to an element of $A$ which corresponds to $\lambda$ in $X^{*}(\hat{A})$, where $F(t, f)=\mu^{-1}(t)$. Since the support $S$ of $F(t, f)$ is open and closed in $G$ we assume that $f$ vanishes outside $S$, namely by a regular function we mean from now on such function which is supported on $S$.

As in the twisted case it is clear that we have the following

Lemma 5. For any irreducible G-module $\pi$ and regular function $f$ the trace $\operatorname{tr} \pi(f)$ is zero unless the Jacquet module $\pi_{U}$ of $\pi$ with respect to the Borel subgroup contains a character $\eta$ with $\eta(t)=\mu(t)$ on $t$ in $N A^{\prime}(R)$, in which case there is a subset $W\left(\pi_{U}\right)$ of $W(A)$ such that

$$
\operatorname{tr} \pi(f)=\sum_{w} \lambda(w \eta)
$$

again $\eta$ is regarded here as an element of $\hat{A}$.

Remark. If $\phi$ in $C\left(G^{\prime}\right)$ and $f$ in $C(G)$ are regular and associated with a character $\mu$ of $A$ and $\lambda$ in $X^{*}(\hat{A})$, then they are matching (we need to compare their orbital integrals only at $a$ in $A^{\prime}$ with $N a$ in $A$ which corresponds to $\lambda$ in $X^{*}(A)$, where we have the equality by definition of regularity).

\section{$\S 5$. Stabilization}

If $G=G L(n)$ then the contents of this section are trivial; the reader who is interested in this special case only may continue directly to the next section. 
Suppose that $E / F$ is a cyclic extension of global fields, and $\sigma$ generates the galois group $\mathrm{Gal}(E / F) . \quad \boldsymbol{G}$ is a reductive connected $F$-group, $\boldsymbol{G}^{\prime}=$ $\operatorname{Res}_{E / F} \boldsymbol{G}$ and $\boldsymbol{G}^{\prime \prime}=\boldsymbol{G}^{\prime} \times\langle\tilde{\sigma}\rangle$ (see Section 1). By $C\left(G^{\prime}\right)$ we denote the set of functions $\phi=\otimes \phi_{v}$ on $G^{\prime}(A)$, supported on $G^{\prime}(A) \times \sigma$, so that each component $\phi_{v}$ lies in $C\left(G_{v}^{\prime}\right)$ (see Section 1) and $\phi_{v}=\phi_{v}^{0}$ for almost all $v$ (see Section 2). Recall the following

Definition. The elements $x, x^{\prime}$ of $G^{\prime}$ are called (stably) $\sigma$-conjugate if $x \times \tilde{\sigma}, x^{\prime} \times \tilde{\sigma}$ are (stably) conjugate, namely there is $y$ in $G^{\prime}$ (resp. $G^{\prime}(\bar{F})$ ) with $y(x \times \tilde{\sigma})=\left(x^{\prime} \times \tilde{\sigma}\right) y$. Here $\bar{F}$ is a separable closure of $F$.

Suppose that $x$ is $\sigma$-regular, namely that the $\sigma$-centralizer $T$ of $x$ in $G^{\prime}$, which is isomorphic to the centralizer of $N x$ in $G$, is a torus. If $x^{\prime}$ lies in $G^{\prime}$ and $y x=x^{\prime} \tilde{\sigma}(y)$ with $y$ in $G^{\prime}(\bar{F})$, since $\tau x=x$ for all $\tau$ in Gal $(\bar{F} / F)$, we have $\tau(y) x=x^{\prime} \tilde{\sigma}(\tau y)$. Let $\tilde{B}^{\prime}(T / F)$ denote the set of $\sigma$ conjugacy classes within the stable $\sigma$-conjugacy class of $x$. Let $B^{\prime}(T / F)$ denote the kernel of the natural map from the group $H^{1}(F, T)$ to the pointed set $H^{1}\left(F, G^{\prime}\right)$. We clearly have the following

Lemma 1. The map which associates to the element $x^{\prime}=y x \tilde{\sigma}\left(y^{-1}\right)$ in the stable $\sigma$-conjugacy class of $x$ the class of the cocycle $\left\{\tau \mapsto y_{\imath}=y^{-1} \tau(y)\right\}$ in $H^{1}(F, T)$, induces a well-defined bijection from $\tilde{B}^{\prime}(T / F)$ to $B^{\prime}(T / F)$.

We shall be interested in the following

Lemma 2. If $H^{1}(E, G)$ is a one point set then so is $H^{1}\left(F, G^{\prime}\right)$, and consequently $B^{\prime}(T / F)=H^{1}(F, T)$.

Proof. We have to compute $H^{1}\left(\mathrm{Gal}(E / F), G^{\prime}(E)\right)$, namely to find the $x=\left(x_{1}, \cdots, x_{e}\right)$ in $G(E) \times \cdots \times G(E)$ with $N x=x \sigma(x) \cdots \sigma^{e-1}(x)$ equals 1. Such $x$ satisfies $x_{1} \sigma\left(x_{2}\right) \cdots \sigma^{e-1}\left(x_{e}\right)=1$. Hence $u=\left(u_{1}, \cdots, u_{e}\right)$, with $u_{i}=$ $x_{i} \sigma\left(x_{i+1}\right) \cdots \sigma^{e-i}\left(x_{e}\right)$, satisfies $x=u \sigma\left(u^{-1}\right)$, as required.

Remark. More generally, it is clear that if $K / F$ is a finite separable extension and $G$ is any algebraic group over $K$, then $H^{1}\left(F, \operatorname{Res}_{K / F} G\right) \simeq$ $H^{1}(K, G)$; indeed, if $A=\operatorname{Gal}(\bar{F} / F)$ and $B=\operatorname{Gal}(\bar{F} / K)$, and $M=G(\bar{F})$, then $\left(\operatorname{Res}_{K / F} G\right)(\bar{F})$ is the induced module $I_{B}^{A} M$, and $H^{1}\left(A, I_{B}^{A} M\right)=H^{1}(B, M)$.

Definition. (1) If $F$ is a local field, put $\Phi^{\prime}(x, \phi)=\sum_{x^{\prime}} \Phi\left(x^{\prime}, \phi\right)$, where the sum ranges over a set of representatives $x^{\prime}$ for the $\sigma$-conjugacy classes within the stable $\sigma$-conjugacy class of $x$, or, in short, over $\tilde{B}^{\prime}(T / F)$. If $F$ is global and $x$ lies in $G^{\prime}$, then $\Phi^{\prime}\left(x, \phi_{v}^{0}\right)=\Phi\left(x, \phi_{v}^{0}\right)$ for almost all $v$, and we define $\Phi^{\prime}(x, \phi)$ to be $\prod_{v} \Phi^{\prime}\left(x, \phi_{v}\right)$. Denote by $A$ the ring of adeles of $F$. 
(2) Put $H^{\prime}(A, T)$ for the restricted pointed sum $\oplus H^{1}\left(F_{v}, T\right)$. Put $X *(T)=\operatorname{Hom}\left(G_{m}, T\right)$ for the group of $\bar{F}$-morphisms from the multiplicative group $\boldsymbol{G}_{m}$ to the torus $T$ (one-parameter subgroups). It is a free $Z$ module of finite rank $r$, where $r$ is the rank of $G$. Note that $X *\left(T_{v}\right)=$ $X *(T)$. Let $K$ be a finite galois extension of $F$ over which $T$ splits. Gal $(K / F)$ acts on $T$, hence on $X_{*}(T)$; denote by $N_{K / F}$ the sum of $\tau$ over $\operatorname{Gal}(K / F)$.

The Tate-Nakayama duality [Ta] (or [Se]) implies the following

Lemma 3. The quotient of $H^{1}(A, T)$ by the image of $H^{1}(F, T)$ injects in $H^{-1}(\mathrm{Gal}(K / F), X *(T))$, namely in the quotient

$$
k^{\prime}(T)=\left\{\mu \text { in } X *(T) ; N_{K / F} \mu=0\right\} /\langle\mu-\tau \mu ; \tau \text { in } \operatorname{Gal}(K / F)\rangle .
$$

However, in general, $H^{1}(F, T)$ does not inject in $H^{1}(A, T)$, and the monomorphism from $H^{1}(A, T) / \operatorname{Im} H^{1}(F, T)$ to $H^{-1}(\operatorname{Gal}(K / F), X *(T))$ is not surjective. In the local case, if $K_{v}$ is a finite galois extension of $F_{v}$ over which $T_{v}=T\left(F_{v}\right)$ splits, the theory of [Ta] implies the following

Lemma 4. The group $H^{1}\left(F_{v}, T\right)$ is equal to $H^{-1}\left(\operatorname{Gal}\left(K_{v} / F_{v}\right), X *(T)\right)$, namely to the quotient

$$
k^{\prime}\left(T_{v}\right)=\left\{\mu \text { in } X *(T) ; N_{K_{v} / F_{v} v} \mu=0\right\} /\left\langle\mu-\tau \mu ; \tau \text { in } \operatorname{Gal}\left(K_{v} / F_{v}\right), \mu \text { in } X *(T)\right\rangle .
$$

Proposition 5.1. Suppose that $F$ is global; $u$ is a place of $F ; x$ is (a rational element) in $G^{\prime}$ which is o-elliptic regular in $G_{u^{\prime}}^{\prime}$; it determines $T, K$ as above. Assume that (1) the component $\phi_{u}$ of $\phi$ at $u$ satisfies $\Phi\left(x, \phi_{u}\right)=$ $\Phi\left(x^{\prime}, \phi_{u}\right)$ for any $x^{\prime}$ in $G_{u}^{\prime}$ stably $\sigma$-conjugate to $x$; and (2) that $\mathrm{Gal}\left(K_{u} / F_{u}\right)$ $\simeq \operatorname{Gal}(K / F)$, hence that the representations $\rho_{u}: \operatorname{Gal}\left(K_{u} / F_{u}\right) \rightarrow \operatorname{Aut} Z^{r}, \rho$ : Gal $(K / F) \rightarrow$ Aut $Z^{r}$, which determine $T_{u}, T$, are equivalent. Then

$$
\sum_{x^{\prime}} \Phi\left(x^{\prime}, \phi\right) \text { is equal to }\left[k^{\prime}(T)\right]^{-1} \Phi^{\prime}(x, \phi) .
$$

The sum ranges over $\tilde{B}^{\prime}(T / F)$, namely over a set of representatives for the $\sigma$-conjugacy classes within the stable $\sigma$-conjugacy class of $x$ in $G^{\prime}$.

Proof. It is clear from [Ta] that under the assumption of (2) there is an exact sequence

$$
0 \rightarrow H^{1}(F, T) \rightarrow H^{1}(A, T) \rightarrow H^{-1}(\mathrm{Gal}(K / F), X *(T)) \rightarrow 0
$$

which splits, and in particular that $H^{1}(A, T) / H^{1}(F, T)=H^{1}\left(F_{u}, T_{u}\right)$. Since (1) asserts that $\Phi\left(x, \phi_{u}\right)$ is constant on $\tilde{B}^{\prime}\left(T_{u} / F_{u}\right)$, it follows that $\sum_{x^{\prime}} \Phi\left(x^{\prime}, \phi\right)$ (sum over $x^{\prime}$ in $\tilde{B}(T / F)$ ) is constant on $\tilde{B}^{\prime}(T / A)$, and the proposition follows. 
Remark. (1) Proposition 5.1 is stated and proved when $H^{1}(E, G)=$ $H^{1}\left(F, G^{\prime}\right)$ is trivial; it can be stated and proved in general, as is done in the non-twisted case in Proposition 5.2, but we do not do it here. As an example, when $E / F$ is quadratic and $G$ is the unitary group $U(n, E / F)$ whose $F$-points are the $g$ in $G(E)=G L(n, E)$ with $\sigma(g)=J^{t} \bar{g}^{-1} J^{-1}$ equals $g$, then $H^{1}(E, G)=H^{1}(\mathrm{Gal}(\bar{F} / E), G L(n, \bar{F}))$ is trivial, but $H^{1}(F, G)$ is nontrivial even when $n=1$.

(2) If $G^{\prime}$ is semi-simple and simply-connected, then $H^{1}\left(F_{v}, G^{\prime}\right)$ is trivial when $F_{v}$ is a non-archimedean local field, or $F_{v}=C$. The Hasse principle asserts that $H^{1}\left(F, G^{\prime}\right.$ ) is isomorphic (as a pointed set) to the restricted pointed direct sum $\oplus_{v} H^{1}\left(F_{v}, G^{\prime}\right)$; for our $G^{\prime}$ this sum ranges over the real places only. Hence $H^{1}\left(F, G^{\prime}\right)$ is trivial if $F$ has no real places (it is totally imaginary if its characteristic is zero).

We also have to deal with the non-twisted case of the connected reductive $F$-group $G$, where $F$ is global. Here $x^{\prime}$ and $x$ in $G$ are called (stably) conjugate if there is $y$ in $G$ (resp. $G(\bar{F})$ ) with $x^{\prime}=y^{-1} x y$. The map $y \mapsto\left\{\tau \rightarrow y_{\imath}=y^{-1} \tau(y)\right\}$ defines an isomorphism from the set $\tilde{B}(T / F)$ of conjugacy classes within the stable conjugacy class of $x$ in $G$ to the kernel $B(T / F)=\operatorname{ker}\left[H^{1}(F, T) \rightarrow H^{1}(F, G)\right]$ of the natural map from the group $H^{1}(F, T)$ to the set $H^{1}(F, G)$. Let $G_{\mathrm{sc}}$ be the semi-simple simply-connected covering group of the derived group $G_{\text {der }}$ of $G$. Since $y$ can be taken to be in $G_{\mathrm{der}}$, and it can be lifted to $G_{\mathrm{sc}}$, it is clear that $B(T / F)$ is the image in $H^{1}(F, T)$ of $B\left(T_{\mathrm{sc}} / F\right)=\operatorname{ker}\left[H^{1}\left(F, T_{\mathrm{sc}}\right) \rightarrow H^{1}\left(F, G_{\mathrm{sc}}\right)\right]$, where $T_{\mathrm{sc}}$ is the inverse image of $T$ in $G_{\mathrm{sc}}$. Let $C(T / F)$ be the image $\operatorname{Im}\left[H^{1}\left(F, T_{\mathrm{sc}}\right) \rightarrow H^{1}(F, T)\right]$ of the natural map from $H^{1}\left(F, T_{\text {sc }}\right)$ to $H^{1}(F, T)$. It is a group, and $B(T / F)$ is a subset of $C(T / F)$. This discussion is valid also when $F$ is replaced by a local field $F_{v}$. We put $C(T / A)$ for the restricted pointed direct sum $\oplus C\left(T / F_{v}\right)$, and note that by [Ta] the quotient $C(T / A) / \operatorname{Im} C(T / F)$ embeds in

$$
k(T)=\left\{\mu \text { in } X *\left(T_{\mathrm{sc}}\right) ; N_{K / F} \mu=0\right\} /\langle\mu-\tau \mu ; \tau \text { in Gal }(K / F), \mu \text { in } X *(T)\rangle .
$$

Moreover, $C\left(T / F_{v}\right)$ is isomorphic to the local analogue $k\left(T_{v}\right)$. We write $\Phi\left(x^{\prime}, f\right)=0$ for $x^{\prime}$ in $C(T / F)-B(T / F)$, locally and globally. As usual, for $x$ in $G$ we write $\Phi^{\prime}(x, f)$ for the product of the local sums $\Phi^{\prime}\left(x, f_{v}\right)$; the product is absolutely convergent since $\Phi^{\prime}\left(x, f_{v}^{0}\right)=\Phi\left(x, f_{v}^{0}\right)$ for almost all $v$. We have

Proposition 5.2. Suppose that $F$ is global; $u$ is a place of $F ; x$ in $G$ is elliptic regular in $G_{u}$. Assume that (1) the component $f_{u}$ of $f$ at $u$ 
satisfies $\Phi\left(x, f_{u}\right)=\Phi\left(x^{\prime}, f_{u}\right)$ for any $x^{\prime}$ in $G_{u}$ stably conjugate to $x$; (2) the assumption (2) of (5.1) holds. Then

$$
\sum_{x^{\prime}} \Phi\left(x^{\prime}, f\right) \text { is equal to }[k(T)]^{-1} \Phi^{\prime}(x, f) .
$$

The sum ranges over the $x^{\prime}$ in $C(T / F)$.

The proof is the same as in the twisted case.

For the comparison of the sums over $x$ in the trace formulae we fix a differential form of highest degree on $\boldsymbol{G}$ defined over $F$. It defines Haar measures $d^{\prime} x_{v}$ and $d x_{v}$ on $G_{v}^{\prime}$ and $G_{v}$ at all places $v$. The Tamagawa measures $d x=\otimes d x_{v}$ on $G(A)$ and $d^{\prime} x=\otimes d^{\prime} x_{v}$ on $G^{\prime}(A)$ are independent of the choice of the $F$-rational differential form on $G$, and they satisfy $\left|G^{\prime}\right|$ $G^{\prime}(A)|=\delta| G \backslash G(A) \mid$, where $\delta$ is the index of $k(T)$ in $k^{\prime}(T) ; T$ is a torus which is elliptic at $u$, and $\delta$ is independent of the choice of $T$.

\section{$\S 6$. Comparison}

Notations as in Section 5. We first record a consequence of Arthur's work [A], generalized to the twisted case by Clozel, Labesse, Langlands (seminar at IAS, 1984).

Lemma 0. Suppose the components at $u_{i}(0 \leq i \leq r)$ of the function $\phi$ in $C\left(G^{\prime}\right)$ are supported on the $\sigma$-elliptic regular set of $G_{u_{i}}^{\prime}$, and $r$ is larger then or equal to the rank of $G$. Further $\phi_{u_{1}}$ vanishes at the $x$ in $G_{u_{1}}^{\prime} \times \sigma$ for which there are $g$ in $G_{u_{1}}^{\prime}$ and $z \neq 1$ in $Z\left(G_{u_{1}}^{\prime}\right)$ with $g^{-1} x g=z x$. Then

$$
\sum_{x}\left|Z^{\prime}(A) T \backslash T(A)\right| \Phi(x, \phi)=\sum_{\pi^{\prime}} c_{\pi^{\prime}} \operatorname{tr} \pi^{\prime}(\phi) .
$$

The sum over $x$ is finite. It ranges over the $\sigma$-conjugacy classes of $\sigma$-regular $x$ in $G^{\prime}$ which are o-elliptic at the $u_{i} . T$ is the centralizer of $N x$ in $G$. The sum over $\pi^{\prime}$ is abosolutely convergent. It ranges over $\sigma$-invariant automorphic $G^{\prime}$-modules. The $c_{\pi^{\prime}}$ are complex numbers.

Remark. The lemma holds also in the case of $G$, where $e=1$ and $\sigma=1$.

Proof. The assumption at $u_{1}$ alone implies that the sum $\sum J_{o}(f)$ of [A] is equal to our sum over $x$. It is well known that it is finite; for a proof see, e.g., [Rig], I, Section 3. The sum $\sum J_{x}(f)$ consists of integrals of logarithmic derivatives of intertwining operators acting on induced representations. As the degree of the derivatives is at most $\mathrm{rk}(G)$, our 
$r+1$ assumptions imply the vanishing of all integrals. There remains a discrete sum of irreducible representations $\pi^{\prime}$, whose components at $u_{i}$ are $\sigma$-elliptic. They are not necessarily square-integrable (cf. case of $S L(2)$ ). Since $\pi^{\prime}$ is irreducible, the intertwining operator is constant and the $c_{\pi}$, is its value. It is integral and positive for cuspidal $\pi^{\prime}$.

Remark. (1) We use Lemma 0 in the proof of the Proposition below. However, when $G_{u}$ is a split local group it suffices (clearly when $G=$ $G L(n)$, and by the arguments of $[\mathrm{K}]$, appendix, when $G \neq G L(n)$, to use the simple trace formula (of Deligne-Kazhdan (see [Rig], I, Section 3)), where at some place $v$ of $F$ which splits in $E$ we take a supercusp form $f_{v}$. For a non-split local group $G_{u}$ and elliptic regular $\gamma_{u}$ in $G_{u}$ there may not exist a global field $F$, group $G$ over $F$, element $\gamma$ in $G$ near $\gamma_{u}$ in $G_{u}$, such that $\gamma$ is elliptic in $G_{v}$, where $v$ is a place of $F$ which splits in $E$. If $v$ is a place of $F$ which stays prime in $E$ it is not clear that there exists a $\sigma$-invariant supersuspidal $G_{v}^{\prime}$-module. Hence, without further work we cannot use the trace formula with a supercuspidal component in the quasisplit case, and have to use Lemma 0.

In the proof of the Proposition below, we shall use the following

Remark. (2) If the place $v$ splits in $E / F$, given $f_{v}$ in $C\left(G_{v}\right)$ (which is right $C_{v}$-invariant, where $C_{v}$ is a compact open subgroup of $G_{v}$ ), we take a measure $f_{v}^{\prime}$ of volume one with $f_{v} * f_{v}^{\prime}=f_{v}$ (namely the quotient by the volume $\left|C_{v}\right|$ of the characteristic function of $\left.C_{v}\right)$. Then $\phi_{v}=\left(f_{v}, f_{v}^{\prime}, \cdots, f_{v}^{\prime}\right)$ in $C\left(G_{v}\right)$ satisfies $\Phi\left(x, \phi_{v}\right)=\Phi\left(N x, f_{v}\right)$ and $F\left(x, \phi_{v}\right)=F\left(N x, f_{v}\right)$. Moreover, given a $G_{v}$-module $\pi_{v}$ we have $\operatorname{tr} \pi_{v}^{\prime}\left(\phi_{v}\right)=\operatorname{tr} \pi_{v}\left(f_{v}\right)$, where $\pi_{v}^{\prime}$ is the $G_{v}^{\prime}=G_{v} \times \cdots \times$ $G_{v}$-module $\pi_{v} \times{ }^{\sigma} \pi_{v} \times \cdots \times{ }^{\sigma e-1} \pi_{v}$; see [GL(3)], Section 1.5.2.

The following is the main result of our paper. It is the crucial step in the approximation argument needed to derive local and global lifting information from the trace formula.

Proposition. Suppose that $E_{u}$ is an unramified cyclic field extension of $F_{u}$, and $f_{u}$ is the image of the spherical function $\phi_{u}$ under the map $H_{u}^{\prime} \rightarrow H_{u}$ which is defined in Section 2 ; namely $\phi_{u}$ corresponds to $f_{u}$. Then $F^{\prime}\left(x, \phi_{u}\right)=F^{\prime}\left(N x, f_{u}\right)$ for all $\sigma$-regular $x$ in $G_{u}^{\prime}$.

Proof. This has been shown for $x$ in $A_{u}^{\prime}=A\left(E_{u}\right)$ in Section 2. Using a standard change of variables formula of the form $F\left(x, \phi_{u}\right)=F^{M}\left(x, \phi_{u N}\right)$ ( $x$ regular, in $M=M_{u}$ ), we may assume that $N x$ is elliptic regular in $G_{u}$. 
For simplicity we denote $E_{u}, F_{u}, f_{u}, \phi_{u}, G_{u}, G_{u}^{\prime}$, by ' $E,^{\prime} F,^{\prime} f,^{\prime} \phi,^{\prime} G,^{\prime} G{ }^{\prime}$. Denote the centralizer of $N x$ in ' $G$ by ' $T$. It is an elliptic torus. Let ' $K$ be a finite galois extension of ' $F$ over which ' $T$ splits. Then ' $T$ is defined by a representation ' $\rho$ : Gal $\left({ }^{\prime} K /{ }^{\prime} F\right) \rightarrow$ Aut $X *\left({ }^{\prime} T\right)$.

Let $F$ be a global (totally imaginary if the characteristic of ' $F$ is zero) whose completion at its places $u, u_{0}, \cdots, u_{r}$ is the local ' $F$. Let $G$ be a reductive quasi-split group over $F$ with $G_{u}, G_{u_{i}}$ isomorphic to ' $G$ such that $G$ is dense in ' $G$. Let $K$ be a galois extension of $F$ with $K \otimes_{F} F_{u_{t}}$ $=' K$, and $K \otimes_{F} F_{u}={ }^{\prime} K$, with

$$
\mathrm{Gal}(K / F) \simeq \mathrm{Gal}\left({ }^{\prime} K /^{\prime} F\right) .
$$

Let $T$ be a torus of $G$ over $F$, which splits over $K$, such that $T_{u} \simeq$ 'T, $T_{u_{i}} \simeq{ }^{\prime} T$, and $T=T(F)$ is dense in ' $T$. The existence of a pair $(T, G)$ with such properties is proven in [Rig], Lemma I.4. Finally we choose a cyclic extension $E$ of $F$ such that $\mathrm{Gal}(E / F), \mathrm{Gal}\left(E_{u_{i}} / F_{u_{i}}\right)$ and $\mathrm{Gal}\left(E_{u} / F_{u}\right)$ are isomorphic. It is clear that (2) of (5.1) is held at $u_{i}$ by construction.

Choose functions $f$ in $C(G)$ and $\phi$ in $C\left(G^{\prime}\right)$ as follows. At all places $v$ of $F$ we require that $F^{\prime}\left(g, \phi_{v}\right)=F^{\prime}\left(N g, f_{v}\right)$ for all $\sigma$-regular $g$ in $G_{v}^{\prime}$, and $F^{\prime}\left(g, f_{v}\right)=0$ if $g$ is regular in $G_{v}$ but not a norm. The choice of $f, \phi$ is possible by Proposition 3, since for almost all $v$ we take $f_{v}=f_{v}^{0}$ and $\phi_{v}=$ $\phi_{v}^{0}$. At the remaining finite set of places we take $f_{v}$ and $\phi_{v}$ to be supported on the regular sets of $G_{v}$ and $G_{v}^{\prime}$. If $v$ splits in $E / F$ then $f_{v}, \phi_{v}$ are related as in Remark (2) which is stated before the proposition; this holds in particular for the archimedean places. At the places $v=u_{i}(0 \leq i \leq r)$, the components $f_{v}, \phi_{v}$ are taken to be supported on the $(\sigma-)$ regular elliptic set of $G_{v}, G_{v}^{\prime}$, such that $F\left(g^{\prime}, \phi_{v}\right), F\left(g, f_{v}\right)$ are $(\sigma-)$ stable functions. The conditions of Lemma 0 are satisfied, and so do the conditions of (5.1) and (5.2). Finally at $u$ we choose $\mu=1$ and a regular $\lambda$ in $X^{*}(\hat{A})$ as in the Definitions of Section 4. As remarked at the end of Section 4, the corresponding regular functions $f_{u}$ and $\phi_{u}$ are matching. We conlcude the following

Lemma 1. For the functions $f, \phi$ chosen above we have

$$
\sum c_{\pi} \operatorname{tr} \pi(f)=\sum c_{\pi^{\prime}} \operatorname{tr} \pi^{\prime}(\phi)
$$

The sums range over the $\pi$ and $\pi^{\prime}$ which appear in Lemma 0 ; they are absolutely convergent.

Write $F_{\infty}$ for the product of the completions of $F$ at its archimedean 
places, and $G_{\infty}, G_{\infty}^{\prime}$ for $G\left(F_{\infty}\right), G^{\prime}\left(F_{\infty}\right)$. Given irreducible modules $\pi_{\infty}$ and $\pi_{\infty}^{\prime}=\left(\pi_{\infty},{ }^{\sigma} \pi_{\infty}, \cdots,{ }^{e-1} \pi_{\infty}\right)$ of $G_{\infty}$ and $G_{\infty}^{\prime}$, we have $\operatorname{tr} \pi_{\infty}\left(f_{\infty}\right)=\operatorname{tr} \pi_{\infty}^{\prime}\left(\phi_{\infty}\right)$. Put $f^{\infty}=\otimes f_{v}, \phi^{\infty}=\otimes \phi_{v}$ (product over finite $v$ ); $\pi^{\infty}, \pi^{\prime \infty}$ are similarly defined. From a standard statement (see e.g., [Rig]) of "generalized" linear independence of characters for absolutely convergent sums (as are the sums of (6.1)) we deduce the following

Lemma 2. We have

$$
\sum c\left(\pi^{\infty}\right) \operatorname{tr} \pi^{\infty}\left(f^{\infty}\right)=\sum c\left(\pi^{\prime \infty}\right) \operatorname{tr} \pi^{\infty \infty}\left(\phi^{\infty}\right) .
$$

The sums are over the $\pi^{\infty}, \pi^{\prime \infty}$ so that $\pi=\pi^{\infty} \otimes \pi_{\infty}, \pi^{\prime}=\pi^{\prime \infty} \otimes \pi_{\infty}^{\prime}$ appear in (6.1).

We write the last identity in the form

$$
\sum c\left(\pi_{u}\right) \operatorname{tr} \pi_{u}\left(f_{u}\right)=\sum c\left(\pi_{u}^{\prime}\right) \operatorname{tr} \pi_{u}^{\prime}\left(\phi_{u}\right) .
$$

The sums range over (equivalence classes of) $G_{u}$ - and $G_{u}^{\prime}$-modules $\pi_{u}$ and $\pi_{u}^{\prime}$ which appear as components of $\pi^{\infty}, \pi^{\infty}$ in the previous sum, and which have an Iwahori fixed vector (by $\left[\mathrm{B}^{\prime}\right]$, Lemma 4.7; indeed, as noted in Lemma 4.4 , if $\operatorname{tr} \pi_{u}\left(f_{u}\right) \neq 0$, since $\mu=1$ the definition of regular $f_{u}$ implies that the Jacquet module of $\pi_{u}$ with respect to a Borel subgroup has an unramified exponent).

Now given $\pi_{\infty}$, or $\pi_{\infty}^{\prime}$, and a compact open subgroup $C$ of $G\left(A_{f}\right)$ or $G^{\prime}\left(A_{f}\right)$, where $A_{f}$ denotes the ring of finite adeles, there are only finitely many automorphic $G$ - or $G^{\prime}$-modules with a $C$-fixed vector and the given component at $\infty$ (see, e.g., [BJ]). Hence, we obtain the following

LemMa 3. For a fixed choice of $f_{v}$ and $\phi_{v}$ for $v \neq u$, the sums over $\pi^{\infty}, \pi^{\prime \infty}$, hence those over $\pi_{u}, \pi_{u}^{\prime}$, are finite.

Recall that by Lemma 4.3 we have $\operatorname{tr} \pi_{u}\left(f_{u}\right)=\Sigma_{w} \lambda(w \eta)$; the sum ranges over a subset of $W(A)$ depending on $\pi_{u}$. The same formula holds for $\operatorname{tr} \pi_{u}^{\prime}\left(\phi_{u}\right)$ by Lemma 4.5. As $\lambda$ varies in a sufficiently large subset of $X^{*}(\hat{A})$, we can apply linear independence of (finitely many) characters to conclude that we have the following

Lemma 4. For any unramified $\sigma$-invariant character $\eta_{u}^{\prime}$ of $A_{u}^{\prime}$, we have

$$
\sum c\left(\pi_{u}\right) \operatorname{tr} \pi_{u}\left(f_{u}\right)=\sum c\left(\pi_{u}^{\prime}\right) \operatorname{tr} \pi_{u}^{\prime}\left(\phi_{u}\right) .
$$

Here the sum on the right ranges over the (finitely many) $G_{u}^{\prime}$-modules $\pi_{u}^{\prime}$ in the composition series of the induced representation $I\left(\eta_{u}^{\prime}\right)$. The sum on the 
left extends over the $G_{u}$-modules $\pi_{u}$ in the composition series of the induced representation $I\left(\eta_{u}\right)$, where $\eta_{u}$ is any unramified character of $A_{u}$ with $\eta_{u}(N a)=\eta_{u}^{\prime}(a)$

However (6.4) holds not only for regular functions $f_{u}, \phi_{u}$, but also for the unit elements $f_{u}^{0}, \phi_{u}^{0}$ of the Hecke algebras $H_{u}, H_{u}^{\prime}$. We conclude the following

Lemma 5. We have $c\left(\pi_{u}\right)=c\left(\pi_{u}^{\prime}\right)$ whenever $\pi_{u}, \pi_{u}^{\prime}$ are spherical and correspond to each other by the map $\hat{G} \rightarrow \hat{G}^{\prime \prime}$ of Section 2 .

Proof. A $G_{u}$-module induced from an unramified character of the Borel subgroup has in its composition series a unique irreducible unramified subquotient. The same holds for $G_{u}^{\prime}$, as required.

It follows that (6.4) holds with any corresponding $f_{u}, \phi_{u}$ in $H_{u}, H_{u}^{\prime}$. The same is true for (6.3), (6.2) and (6.1), hence we have

$$
\sum[k(\tilde{T})]^{-1}|Z(A) \tilde{T} \backslash \tilde{T}(A)| \Phi^{\prime}(N g, f)=\sum\left[k^{\prime}(\tilde{T})\right]^{-1}|Z(A) \tilde{T} \backslash \tilde{T}(A)| \Phi^{\prime}(g, \phi),
$$

where the components $f_{u}, \phi_{u}$ of $f, \phi$ are spherical and corresponding. The sums are finite, and range over stable regular conjugacy classes in $G$ (which are elliptic regular at the $u_{i}$ ). $\tilde{T}$ is the centralizer of $\mathrm{Ng}$ in $G$. We choose $f^{u}=\otimes f_{v}$ (product over $v \neq u$ ) such that $\Phi^{\prime}\left(N x, f^{u}\right) \neq 0$; since $T$ is dense in $T_{u}$ we may assume that the $x$ of the proposition is rational in $T^{\prime}$, and $T$ is the centralizer of $N x$ in $G$. As the sums over $N g$ are finite we can reduce the support of $f_{\infty}$ to assume that for fixed $f_{u}, \phi_{u}$, the sums over $N g$ range over the element $N x$ only. We conclude that $\Phi^{\prime}(N x$, $\left.f_{u}\right)=\Phi^{\prime}\left(x, \phi_{u}\right)$ for any $\sigma$-regular $x$ in $G^{\prime}$ which is $\sigma$-elliptic in $G_{u}^{\prime}$, hence for all $\sigma$-regular $\sigma$-elliptic $x$ in $G_{u}^{\prime}$, as required.

\section{REFERENCES}

[A] J. Arthur, On a family of distributions obtained from Eisenstein series II: explicit formulas, Amer. J. Math., 104 (6) (1982), 1289-1336.

[AC] J. Arthur, L. Clozel, Base change for $G L(n)$, manuscript (1987).

[BZ] I. Bernstein, A. Zelevinskii, Representations of the group $G L(n, F)$ where $F$ is a non-archimedean local field, Uspekhi Mat. Nauk, 31 (3) (1976), 5-70.

[BZ'] - Induced representations of reductive p-adic groups I, Ann. Sci Ecole Norm Sup., 10 (1977), 441-472.

[B] A. Borel, Automorphic L-functions, Proc. Sympos. Pure Math., 33 (1979), II $27-63$.

[B'] - Admissible representations of a semi-simple group over a local field with vectors fixed under an Iwahori subgroup, Invent. Math., 35 (1976), 233-259.

[BJ] A. Borel, H. Jacquet, Automorphic forms and automorphic representations, Proc. Sympos. Pure Math., 33 (1979), I 189-202. 
[Ca] P. Cartier, Representations of $p$-adic groups: A survey, Proc. Sympos. Pure Math., 33 (1979), I 111-155.

[C] W. Casselman, Characters and Jacquet modules, Math. Ann., 230 (1977), 101105; see also: P. Deligne, Le support du caractère d'une représentation supercuspidal, CRASP, 283 (1976), 155-157.

[D] G. van Dijk, Computations of certain induced characters of $p$-adic groups, Math. Ann., 199 (1972), 229-240.

[U(2)] Y. Flicker, Stable and labile base change for $U(2)$, Duke Math. J., 49 (1982), 691-729.

[GL(3)] - The trace formula and base change for $G L(3)$, Springer Lecture Notes, 927 (1982).

[GL(n)] - - On twisted lifting, Trans. Amer. Math. Soc., 290 (1985), 161-178.

[UP] —- Unitary quasi-lifting; preparations, Contemp. Math., 53 (1986), 119-139.

[UA] — Unitary quasi-lifting: applications, Trans. Amer. Math. Soc., 294 (1986), 553-565.

[Rig] - Rigidity for automorphic forms: I. Harmonic analysis; II. Comparison; III. Representations of simple algebras; IV. Automorphic forms on compact unitary groups; J. d'Analyse Math., 48 (1987).

[Sym] —- On the Symmetric Square; I. Definitions and lemmas; II. Applications of a trace formula; III. Orbital integrals, Math. Ann., (1987) ; IV. Twisted trace formula; V. Unstable local transfer (with D. Kazhdan) ; VI. Total global comparison; preprints, Harvard 1986.

[RTF] - Regular trace formula and base-change lifting, Amer. J. Math., (1988).

[FK] Y. Flicker and D. Kazhdan, Metaplectic correspondence, Publ. Math. IHES, 64 (1987), 53-110.

[STF] —- A simple trace formula, J. d'Analyse Math., 48 (1987).

[G] M. Greenberg, Schemata over local rings II, Ann. of Math., 78 (1963), 256-266.

[K] D. Kazhdan, Cuspidal geometry on p-adic groups, J. d'Analyse Math., 47 (1987), 1-36.

[K'] - On lifting, in Springer Lecture Notes, 1041 (1984), 209-249.

[Ko] R. Kottwitz, Rational conjugacy classes in reductive groups, Duke Math. J., 49 (1982), 785-806.

[Ko'] - Base change for unit elements of Hecke algebras, Compositio, Math., 60 (1986), 237-250.

[R] R. Rao, Orbital integrals in reductive groups, Ann. of Math., 96 (1972), 505510.

[Se] J. P. Serre, Corps locaux, Hermann, Paris.

[Ta] J. Tate, The cohomology groups of tori in finite Galois extensions of number fields, Nagoya Math. J., 27 (1966), 709-719.

[Ti] J. Tits, Reductive groups over local fields, Proc. Sympos. Pure Math., 33 (1979), I, 29-69.

Department of Mathematics

Harvard University

Science Center

One Oxford Street

Cambridge, $M A 02138$ 